\title{
Augmenting Curved Robot Surfaces with Soft Tactile Skin
}

\author{
Gereon Buescher, Martin Meier, Guillaume Walck, Robert Haschke and Helge J. Ritter \\ \{gbuescher,mmeier,gwalck,rhaschke,helge\}@techfak.uni-bielefeld.de \\ Neuroinformatics Group, Center of Excellence Cognitive Interaction Technology (CITEC), \\ Bielefeld University, Germany
}

\begin{abstract}
We present a novel, soft, tactile skin composed of a fabric-based, stretchable sensor technology based on the piezoresistive effect. Softness is achieved by a combination of a soft silicone padding covered by a skin of more durable, tearproof silicone with an imprinted surface pattern mimicking human glabrous skin, found e.g. in fingertips. Its very thin layer structure (starting from $2.5 \mathrm{~mm}$ ) facilitates integration on existing robot surfaces, particularly on small and highly curved links. For example, we augmented our Shadow Dexterous Hand with 12 palm sensors, and 2 resp. 3 sensors in the middle resp. proximal phalanges of each finger.

To demonstrate the usefulness and efficiency of the proposed sensor skin, we performed a challenging classification task distinguishing squeezed objects based on their varying stiffness.
\end{abstract}

\section{INTRODUCTION}

Augmenting robot hands and complete robot surfaces with a soft, tactile-sensitive skin is an active research topic promising many advantages for manipulation and safe human-robot interaction. Mimicking soft flesh using elastomer pulps [1] increases compliance, friction, contact area, and thus also grasp stability and the amount of applicable wrenches.

There exists a large body of work considering tactile skin and we can only refer to few of them. [2] utilizes PUfoam as soft flesh for a whole robot body with integrated LED-based tactile sensors. However, the thickness of the skin $(20 \mathrm{~mm})$ impedes the integration on small structures like robot hands. The tactile skin developed for the iCub robot consists of triangle-shaped modules based on flex-PCB for the body [3] and specially folder flex-PCB for the fingertips [4]. Flex-PCBs with even higher spatial resolution of the tactile elements are proposed in [5] and [6]. The major limitation of flex-PCB based sensors, however, is that they are inherently stiff and only bend along 1D surfaces and hence cannot be applied for too small structures.

Tactile sensors based on elastic substrates overcome this limitation. [7] employs a highly stretchable material (150\%), measuring deformations due to applied forces with electrical impedance tomography, a computationally rather costly approach. [8] describes a sensor array based on piezoresistive rubber with an embedded electrode matrix, thus achieving high spatial resolution. A combination of compliant padding and tactile sensing was developed for the BioTac ${ }^{\circledR}$ sensor [9]

This work is supported by the DFG Center of Excellence EXC 277: Cognitive Interaction Technology (CITEC) and has received funding from the EU FP7/2007-2013 grant agreement nr. 601165 of the project WEARHAP. We would like to thank Statex plc. for providing conductive fabric samples.

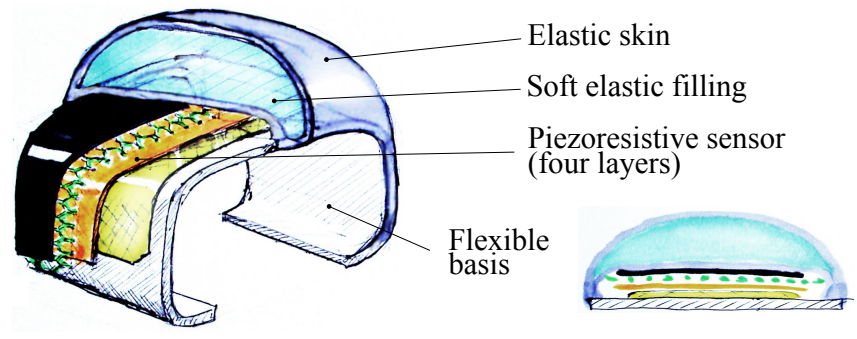

Fig. 1. Cross section through a sensor unit: A flexible basis allows quick mounting, the fabric-based sensor allows for covering complex curved shapes, and the two-component padding unites soft grip and robustness.

providing excellent sensitivity and resolution. However, due to its bulky electronics, integration e.g. on the Shadow robot hands [10] requires to replace a finger link, thus loosing a DoF.

In contrast, the proposed sensor technology is flexible and thin enough to be integrated on existing robot hardware. Particularly, we strove for augmenting the Shadow robot hands with a soft, tactile skin all over the palmar surface. However, the developed technology can be applied in a similar fashion to other robotic hands or arms as well. In any case, we attempt to augment existing robot hardware with tactile sensors, although the manufacturer didn't prepared the robot appropriately. Consequently, there is typically only very little or no space at all available for integration of sensing elements, acquisition electronics, and wiring. Furthermore, curved robot surfaces require flexible rather than rigid sensing units.

In this work we draw on our previous results developing a fabrics-based tactile sensor [11], whose working principles will be shortly summarized for completeness in the next section before we describe its integration into a soft skin for robots. Subsequently, we evaluate the sensor characteristics before considering an application example utilizing the sensors to classify the stiffness of grasped objects.

\section{SENSOR UNIT}

To augment curved robot surfaces with a tactile-sensitive skin we employ our fabric-based sensing technology originally developed for a highly flexible human data glove [11]. The sensing elements (taxels) are composed of several layers of conductive and piezoresistive fabric, having an overall thickness of only $1.5 \mathrm{~mm}$. The piezoresistive layer is sandwiched between two highly conductive layers used as measuring electrodes. Two major effects contribute to 


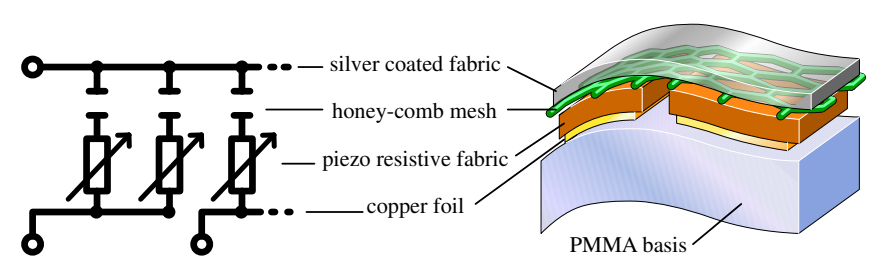

Fig. 2. Illustration of the sensor's working principle: Applied pressure i) increases the contact area between conductive layers, creating new shortcuts, and ii) reduces the resistance of the piezoresistive fabric layer.
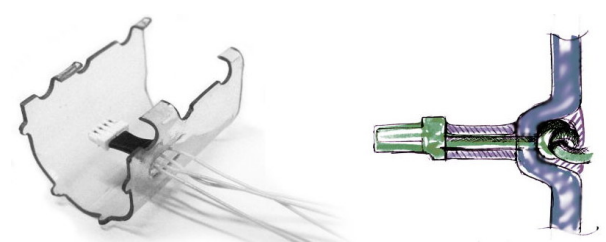

Fig. 3. Support layer of proximal finger phalanx unit with integrated strain relief and assembled connector. Right: Cross section drawing of strain relief.

measured changes of resistance: On the one hand, the piezoresistive fabric causes a direct change of its resistance due to a compression of its semiconductive threads. On the other hand, pressure increases the overall surface contact area between fabric layers, thus establishing new conductive bridges. Both effects are nonlinear and superimpose. Figure 2 schematically summarizes these effects.

While the tactile data glove needed to be flexible and stretchable in all sensor layers, the mounting on a rigid robot surface allows to replace the lowest fabric electrode layer by a rigid material that is directly attached to the robot. However, to facilitate robot repairs and sensor replacements, an important design constraint was to develop a mounting interface that allows for quick mounting and un-mounting of the sensor. To this end, we decided for an almost rigid support basis that will be clicked onto the robot links. On top of this support layer, the sensor layers are glued, followed by a soft elastic skin formed from a silicon rubber pulp. Figure 1 illustrates this overall structure. In the following the individual components will be described in detail.

\section{A. Support Basis and Mounting}

A robust foil of acrylic glass (PMMA) with $0.5 \mathrm{~mm}$ thickness is chosen as the support basis of each sensor unit (Fig. 4a). This basis handles the fixation of the sensor on the robot limbs, the distribution of tension stresses arising in the elastic parts, and it serves as a PCB-like construction ground. The foil is laser cut and bent in hot air using a 3D-printed positive template.

For easy mounting the support layers are equipped with small flaps that clasp on the back of the palm and fingers. The flaps in turn are pinched between the fingers and their covers or fixated using narrow Velcro ${ }^{\circledR}$ stripes on the palm. To mount the units, the PMMA basis will be clicked onto the robot links, slightly bending them to fit over the limbs. Middle and proximal units have their electrical connector built into the inner side of the flanges as depicted in Fig. 3 .

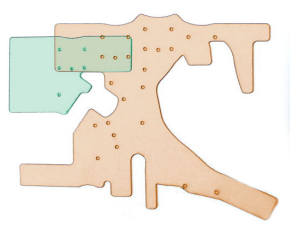

(a) PMMA base material

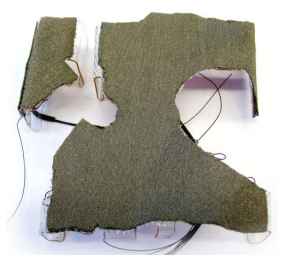

(d) top electrode layer

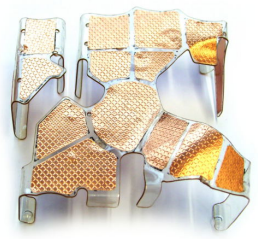

(b) copper foil taxels

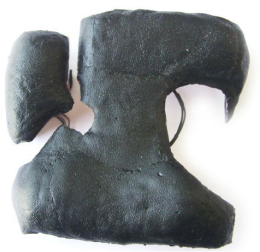

(e) silicone padding

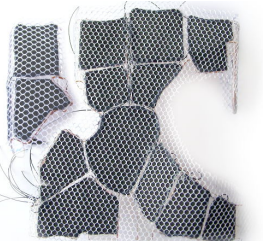

(c) piezoresistive layer

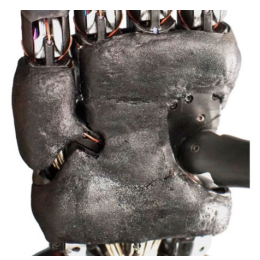

(f) mounted palm sensor
Fig. 4. Intermediate assembly steps of palm sensor unit. (a) laser cut PMMA sheet, (b) copper foil electrodes stuck on heat-bent sheets, (c) added piezoresistive layer and mesh, (d) top fabric electrode layer, (e) soft silicone padding coated with fingerprint pattern, (f) integration on Shadow hand.

The connector can be guided with tweezers for insertion. As the corresponding sockets are hidden deep inside the finger links, removing the sensor units requires to pull on the sensor cables to disconnect them. For this reason, a cable strain relief was integrated within the support layer for those sensors as illustrated in Fig. 3

\section{B. Sensor Layers}

The sensor is built from a piezoresistive fabric layer sandwiched between to highly conductive electrode layers. In contrast to the original, purely fabric-based tactile glove design [11], we here use a self-adhesive, thin, and embossed copper foil as the bottom electrode. By dividing this copper layer into several cells, multiple taxels within a single sensor unit can be realized as shown in Fig. 4p.

The top electrode layer is made of silver-coated fabric, which is elastic and flexible to allow for proper recovering after the sensor was pushed. In between the electrode layers is a semi-conductive fabric layer (placed directly above the copper foil) and a non-conductive mesh. The semiconductive fabric exhibits piezoresistive properties due to its thin polymer matrix coating filled with carbon nano particles. For better separation of the sensitive cells, this piezoresistive layer is also individually isolated for each taxel - matching the taxel structure of the copper foil (Fig. 4c). The mesh layer with its honey-comb structure serves as an isolating air film between the conductive layers avoiding spurious contact measurements. To prevent short circuits and to uniformly distribute shear forces, all layers are sparsely glued together along the boundaries of individual taxels (using non-conductive silicone rubber). For even more details regarding the fabric-based sensor design we refer to [11].

\section{Soft Artificial Pulp and Durable Skin}

On top of the sensing layers a soft silicone rubber padding is placed, covered with a fine-structured, more durable silicone rubber skin. The pulp is realized from 2-component platinum-cure silicone rubber (Smooth-On Inc. Ecoflex ${ }^{\circledR}$ 


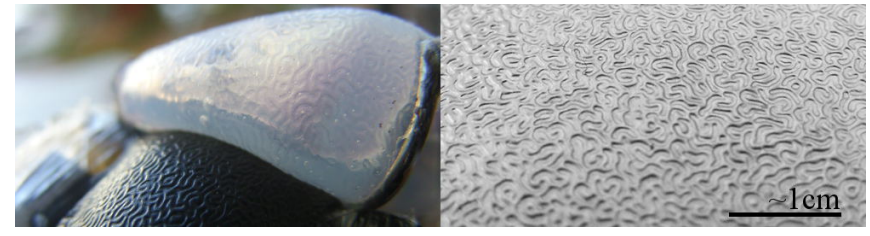

Fig. 5. Left: Photo of the de-molding process. The light material on top is an elastic, patterned mold. Beneath appears the black colored sensor surface. Right: Closeup of the embossed aluminium foil with grooves in size of human fingerprints.

$0010 \mathrm{~A}+\mathrm{B})$ diluted with Ecoflex ${ }^{\circledR}$ Slacker. In an extensive series of tests, the mixing ratio $2 / 2 / 3$ was found to be highly soft, but still elastic and not yet gel-like. The steered and vacuum-vented components are molded in a 3D-printed shell to obtain the desired shapes. As super-soft elastomers will always tend to sweat oil, the pulp needs to be encapsulated to prevent direct contact with the sensor and the surrounding. By gluing the pulp to the sensor using a silicone adhesive an oil barrier is established.

The durable skin is made from single-component silicone rubber, Elastosil ${ }^{\circledR}$ E43. To cover the pulp with this stronger elastomer a silicone mold from Elastosil ${ }^{\circledR}$ E4 was built as depicted in Fig. 5 Its elasticity allows easy de-molding, which prevents the sensor from damage. The surface of the sensing unit is patterned with a fine relief from irregularly arranged, curved grooves with a groove size of ca. $0.5 \mathrm{~mm}$. This correlates to those of human glabrous skin, as found e.g. in fingerprints. The irregular pattern is chosen to prevent directional bias as suggested by [12].

Molding the skin is the last step of sensor production. The E43 therefore is poured into the mold and the sensor unit assembly is pushed in. The E43 fills the thin space between the pulp and the mold as well as all remaining hollows, such that a smooth sensor surface is obtained.

\section{Integration and Data Acquisition on Shadow Robot Hand}

Fortunately, the Shadow robot hands offer a few extension points to integrate additional sensors. Firstly, there are some free analog to digital channels available at different places in the palm and the fingers, which are already sampled by the standard firmware. Secondly, palm and fingers have free SPI bus sockets to connect additional boards. However, in this case a firmware modification is required to access those boards and transfer the data through the EtherCAT ${ }^{\circledR}$ bus to the host. Both possibilities were employed. The finger sensors are directly attached to three resp. two spare AD channels in the proximal resp. middle phalanx. The number of free channels thus directly determined the number and placement of taxels on these units. For the palm, 4 spare AD channels and a ready-to-use auxiliary SPI board, offered by Shadow providing 8 additional channels, are used, summing up to 12 taxels in the palm. The fingertips, providing more space for electronics integration, are equipped with custommade MID fingertip sensors, each providing 12 taxels at higher spatial resolution [13].

While the fingertip sensors are sampled at $750 \mathrm{~Hz}$ with

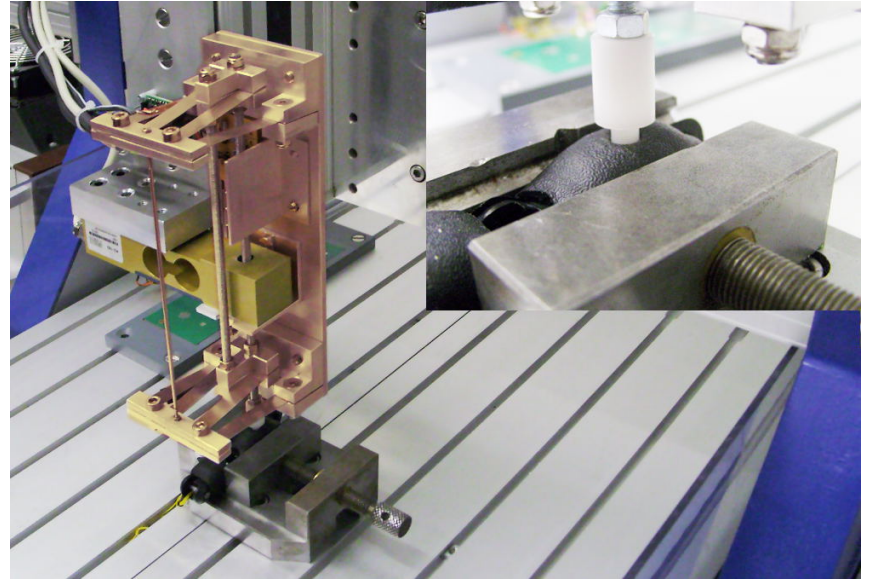

Fig. 6. Evaluation rig with industrial strain gauge (yellow) and suspension (orange). The insert shows a spherical tip probing the proximal phalanx.

10-bit ADC, all other sensors distributed across the finger phalanges and the palm are sampled at $1 \mathrm{kHz}$ with 12-bit ADC. The tactile data transferred to the host PC is published by the Shadow ROS driver over separate ROS topics at $100 \mathrm{~Hz}$ by default. The full $1 \mathrm{kHz}$ update rate is available at the host if needed.

\section{E. Sensor Evaluation}

A custom built measurement rig was used to evaluate the performance of various material compositions for the sensor (Figure 66. The rig consists of 3 perpendicular linear axes with a calibrated industrial strain gauge attached (colored in yellow) to provide gold standard force measurements. To allow for more fine-grained force control, a suspension (colored in orange) is used, transforming fixed-size endeffector motions into spring forces. The probe tips on the end-effector are exchangeable. The sensor characteristic is recorded by automatic loading and unloading sensor samples with forces from 0 to $30 \mathrm{~N}$.

The sensor characteristics of the middle phalanx sensor is depicted in Fig. 7. The curves show the 12-bit sampled sensor output when using spherical plastic probe tips with radii of $2.5 \mathrm{~mm}$ and $12.5 \mathrm{~mm}$ (blue resp. orange curves). Both force response curves show an idle behavior close to small forces, which is due to the high idle resistance deliberately introduced by the spacer layer. First contact is detected around 2-3 N. The curves exhibit the typical hysteresis effect that is innate to piezoresistive elastomers when switching from loading to unloading. Atypical is, that the sensor output even increases slightly in the beginning of the unloading phase.

The smaller probe tip produces a higher slope as can be seen from the blue curve. In previous work, we noticed that the fabric-based tactile sensor operates more like a pressure than a force sensor [11]. However, given the soft flesh of the proposed sensor units, it is impossible to measure the actual contact area and translate force into pressure values in the figure. The repeatability of the sensor is rather good as the 


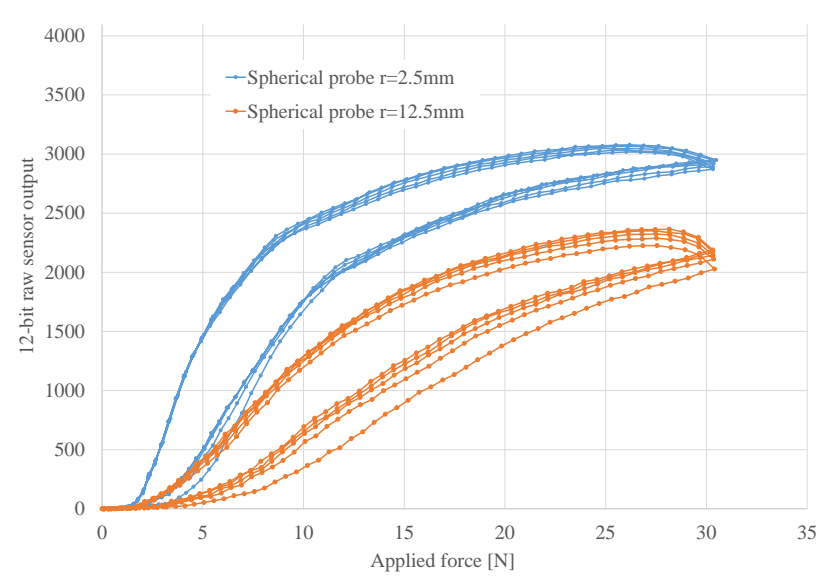

Fig. 7. Characteristics of the proximal phalanx sensor when loading and unloading it 5 times from 0 to $30 \mathrm{~N}$ using differently sized probe tips. The sensor exhibits good repeatability, but also the typical hysteresis effect.

five consecutive loading and unloading curves exhibit a high degree of overlap.

\section{Application: Estimating Object Stiffness}

In order to validate the efficiency of the tactile sensors in a real-world scenario, a preliminary experiment was performed to classify various grasped objects based on their estimated stiffnesses. A recurrent neural network was used to this end.

\section{A. Setup description}

The experiment was performed using our bimanual robot setup employing the Shadow hands fitted with the novel tactile sensors. Only the hands were actively controlled during the experiment, while the arms were moved to an adequate pose in advance.

The hand firmware runs a low-level force control loop (based on tendon forces) to drive the joints of the fingers. All higher-level control loops, e.g. position control, are closed in the host $\mathrm{PC}$, sending tendon force requests to the hand at a rate of $1 \mathrm{kHz}$. The firmware provides only basic maximumforce safety limits, which do not suffice to protect the hand from high loads over longer time. Hence, an improved safety limitation was implemented in the host control loop, based on accumulated tendon force magnitudes for each joint $i$ :

$$
F_{i}^{\mathrm{acc}}(t)=\max \left(0, \int_{0}^{t}\left|F_{i}(t)\right|-F_{\max } d t\right),
$$

where $F_{i}(t)$ is the difference between flexion and extension tendon forces of joint $i$ and $F_{\max }$ a threshold under which the force is acceptable. The maximally allowed force $F_{i}^{\max }(t)$ at time $t$, decreases over time as $F_{i}^{\text {acc }}(t)$ increases:

$$
F_{i}^{\max }(t)=F^{\min }+\left(F^{\mathrm{MAx}}-F^{\mathrm{min}}\right) \cdot \exp \left(-\frac{F_{i}^{\mathrm{acc}}(t)}{\tau}\right) .
$$

Here $F^{\min }$ is the minimal force required for free-space motion and $F^{\mathrm{MAX}}$ is the upper force limit. The exponential decay rate is controlled with the parameter $\tau$, called force endurance. The higher the endurance, the longer the hand can maintain a certain maximum force before saturation limits apply.

For the experiments an endurance value was used that is 4 times higher than the standard value used for object grasping. This allows to apply higher forces required for squeezing the grasped objects. We will refer to the force limiting algorithm when discussing the force plots in Fig. 9 .

\section{B. Experiment protocol}

The experiment protocol is illustrated in Fig. 8. An object was placed in the palm of the hand with the fingers opened (a). Then, the hand was position-controlled towards a power-grasp, the thumb not being involved. As the commanded hand posture would penetrate the object, the force limitation became active, squeezing the object between the palm and the finger phalanges and/or fingertips (b). This grasp was maintained for ca. $10 \mathrm{~s}$ while the force limitation algorithm was reducing tendon forces after a while. Then, the motors were shut off to release tendon forces, allowing the compressed object to slowly recover its original shape and size (c). This recovery phase lasted ca. $5 \mathrm{~s}$ after which the hand was commanded to open the fingers again, completely releasing the object (d). Joint angle and tactile sensor trajectories were recorded at $100 \mathrm{~Hz}$ during the whole experiment duration.

As an example, the data recorded from a single finger for a single trial is shown in Fig. 9. While the top plot shows tactile contact forces along with joint angles (solid vs. dashed curves), the bottom plot displays the effective tendon forces of the two involved actuators (middle and distal joints are coupled). The different phases (A: closing to contact, B: squeezing, C: maintaining, D: recovery, E: releasing) are marked by vertical lines. The most interesting phases for stiffness classification are the squeezing (B) and the shape recovery phase (D). They are clearly separated by events like contact detection, contact force increase, contact force release, and contact loss.

Six different test objects were created using a fabric bag as a cylindrical container, stuffed with various materials of distinguishable stiffness (bubble wrap, soft and hard foam) or deformable properties (set of marbles, and fine or coarse granules) as shown in Fig. 10. A strong cardboard tube served as a seventh, rigid object. All objects have similar shape, i.e. are only distinguished by their stiffness.

\section{Learning}

To classify the objects a Long Short Term Memory Recurrent Neural Network (LSTM) [14] was employed that was

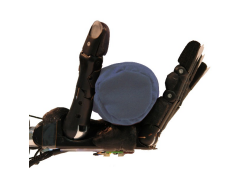

(a) released

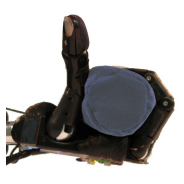

(b) squeezing

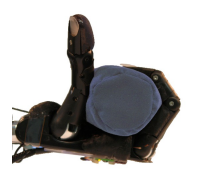

(c) relaxed

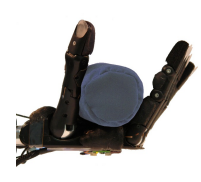

(d) released
Fig. 8. Experiment protocol: robot hand grasping and squeezing an object. 


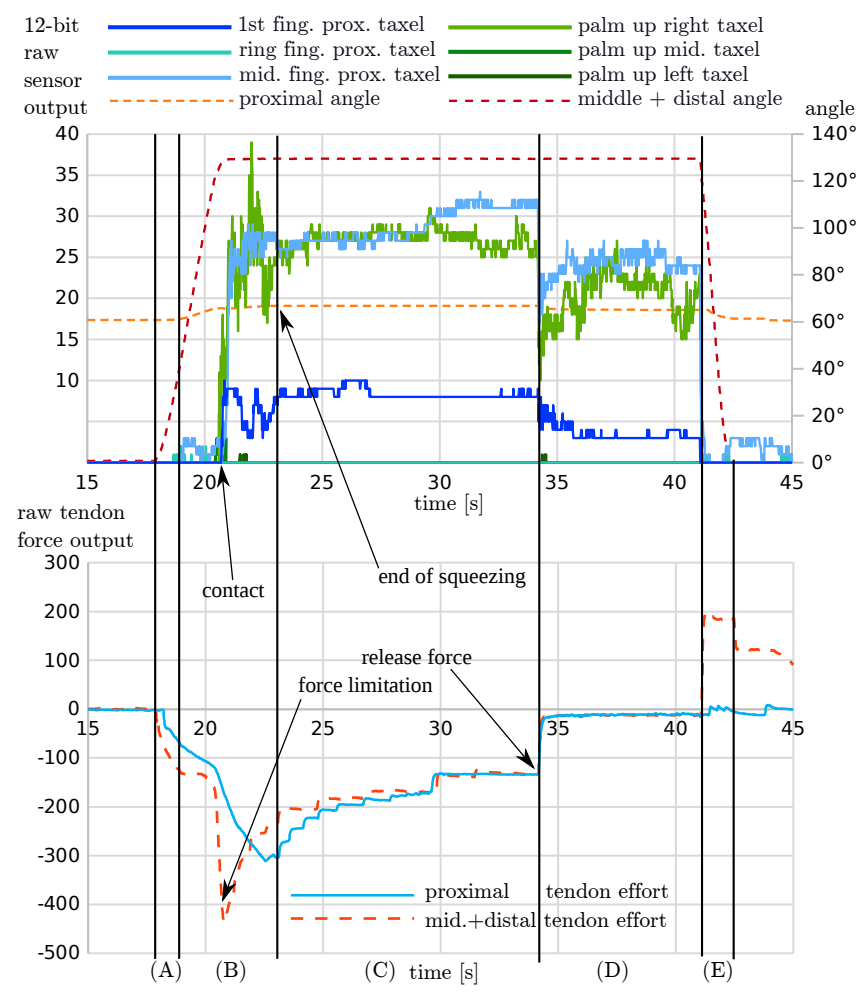

Fig. 9. Plot of tactile data aligned with tendon forces and joint angles during squeezing of a bag filled with Marbles of $1 \mathrm{~cm}$ diameter.

trained on the time series data of various sets of sensor readings as detailed below. LSTM networks have shown very good performance in classifying and predicting time series data, for example in language modeling [15], which motivated the utilization of this specific RNN type in the tactile time series classification task. Initial tests with simpler classification methods like Learning Vector Quantization and Support Vector Machines did not yield useful results.

A single LSTM unit (left in Fig. 11) is composed of a self-coupled neuron with a coupling strength of 1.0 assuring that the neuron can keep its activation $x(t)$ over long time. A multiplicative input gate regulates to which extend network inputs will change the state of the neuron. Similarly an output gate controls to which extend the neuron's own activation is fed back into the network. A forget gate allows to reset the information memorized in the neuron. Hence, the network dynamics updates a single neuron's activation $x$ as follows:

$$
x(t)=y_{f}(t) \cdot x(t-1)+y_{\text {in }}(t) \cdot \sigma\left(\mathbf{w} \cdot \mathbf{x}(t-1)+\mathbf{w}^{u} \cdot \mathbf{u}(t)\right)
$$

The output $y(t)$ is computed by weighting this activation by the output gate's activity:

$$
y(t)=y_{\text {out }}(t) \cdot \sigma(x(t)) .
$$

The gates are implemented as standard neurons as well, whose activity $y_{*}(t)$ is calculated as usual:

$$
y_{*}(t)=\sigma\left(\mathbf{w}_{*} \cdot \mathbf{x}(t-1)+\mathbf{w}_{*}^{u} \cdot \mathbf{u}(t)\right),
$$

where $*$ is a placeholder for subscripts $f$, in, or out. $\mathbf{u}(t)$, $\mathbf{w}_{*}$, and $\mathbf{w}_{*}^{u}$ denote the input at time $t$, and the networks resp.

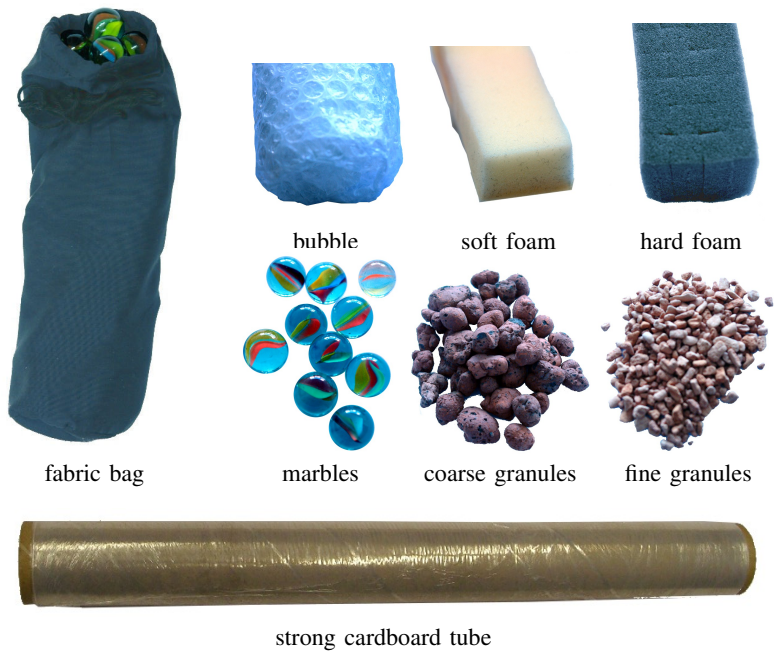

Fig. 10. Test objects: bags fitted with different materials.

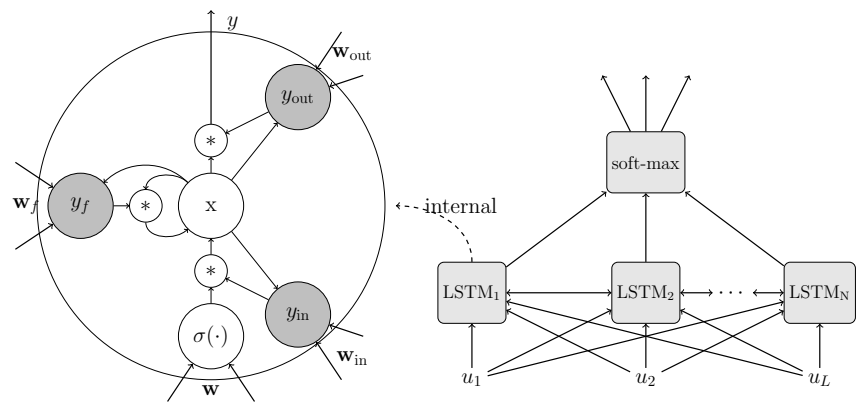

Fig. 11. Illustration of a LSTM unit (left) and its integration into a larger network (right). The recurrent connections are omitted in this schematic, for details please refer to $[14]$.

input weights. The nonlinear squashing function $\sigma$ is applied element-wise to the activation vectors. Commonly used are the logistic function [14] as well as hyperbolic tangent [16].

All LSTM units are recurrently interconnected. The recorded time series data $\mathbf{u}(t)$ is fed as input to all units. For final classification, a linear read-out layer with soft-max normalization is employed.

Training of the LSTM-RNN is done by backpropagation through time (BPTT). Compared to standard backpropagation in feed-forward networks, the recurrent network is (virtually) unfolded in time, transforming the repeated application of the network update rules (eqs. 35 into a sequence of identical feed-forward layers. This unfolded version can be trained by means of standard backpropagation. For details about BPTT, please refer to [17].

\section{Results}

Different network layouts have been evaluated with a varying amount of hidden LSTM units. A network with 160 hidden units proved to be sufficient in all of the following trials up to the 24 dimensional input case, using a learning rate of $10^{-5}$. For the cases with more than 24 dimensional input, the number of LSTM units was increased to 240. In order to assess the relevance of different sensor modalities 


\begin{tabular}{|c|r|l||l|}
\hline$\#$ & \multicolumn{1}{|c|}{ dims } & description & test error \\
\hline \hline 1 & $12-160-7$ & all taxels on palm & $42.23 \%$ \\
2 & $24-160-7$ & all taxels on palm + proximal/middle & $34.58 \%$ \\
3 & $16-160-7$ & 4 central taxels per fingertip & $32.64 \%$ \\
4 & $8-80-7$ & 2 flexion joints per finger & $40.87 \%$ \\
\hline 5 & $20-160-7$ & combination of (1) + (4) & $27.61 \%$ \\
6 & $32-240-7$ & combination of $(2)+(4)$ & $22.06 \%$ \\
\hline 7 & $36-240-7$ & combination of (5) + (3) & $22.43 \%$ \\
8 & $48-240-7$ & combination of (6) + (3) & $20.76 \%$ \\
\hline \multicolumn{2}{|c|}{ random chance } & $85.71 \%$ \\
\hline
\end{tabular}

TABLE I

CLASSIFICATION ERRORS OBTAINED FOR DIFFERENT INPUT DATASETS

for the classification result, different combinations of the available sensor streams were evaluated as input to the network with respect to the achievable classification error.

The multi-dimensional time series, with a length of ca. 3300 steps, was provided to the network one at a time as is, only normalizing each individual sensor channel to the range $[0,1]$. For each of the objects in Fig. 10,6 trials were recorded and split into training, validation and test set (4-1-1).

The classification task is rather challenging, because the time series data are very sparse, exhibiting many zero values for individual tactile cells. This is because only a small subset of taxels actually have contact at the same moment in time and because the sensitivity of the tactile sensor units is still rather limited considering the restricted amount of force the robot hand can actively exert. The latter can be seen from the small exploited output range (0-40 units) in Fig. 9. which is only $1 \%$ of the whole ADC range. Additionally, the materials used for the test objects are highly similar, for example considering the soft foam and the bubble wrap, or the marbles and the large granules.

The finally achieved test errors after training are summarized in Table 1 Random guessing for this classification task would result in an error rate of $85.7 \%$ - corresponding to the probability of $1 / 7$ for guessing correctly.

Using individual sensor modalities only (1-4), the network is already able to produce classification results clearly above chance level. The fingertip sensors (3) and the proposed tactile sensors (2) obtain similar results, although the former are currently much more sensitive (using a different sensor technology [13]). Thus, the sensitivity of the proposed sensors was sufficient for this real-world task.

Combining different sensor modalities naturally improves the results. As stiffness corresponds to the relation of object penetration and measured forces, we first augmented the tactile sensor input with angular measurements (5-6), which significantly decreased the error, but required a larger network. Incorporating tactile data from the more sensitive fingertip sensors (7-8), improved the results by a few more percents. To this end, we considered the four central taxels on the fingertips, neglecting the other ones, directed sideways and never exhibiting contact.

Obviously, the more input data is provided, the better the network can extract the relevant information from the time series, finally achieving a classification error close to $20 \%$.

\section{CONCLUSION}

In this paper, we presented the design and detailed building steps of a novel, soft, and tactile-sensitive robot skin. Due to its thin design, the skin can be easily augmented to existing robot hardware, particularly also in narrow spaces and on highly curved surfaces. We reported on the integration of these sensors in the palm and finger phalanges of the Shadow Robot Hands providing them with a sense of touch not only in the fingertips but all over the palmar surface.

An example application, classifying various objects from their differently perceived stiffness properties, proved the usefulness and efficiency of the proposed sensor skin for a challenging, real-world task. Even though only a small fraction of the available output range of the sensors was exploited in this experiment, the sensor's sensitivity was sufficient to achieve promising classification results.

In future work, we will further improve the sensor's sensitivity to first touch by fine-tuning the acquisition electronics and considering other measurement principles, e.g. measuring changes in capacity of the sensor units. Further, we will consider to reverse the layer structure of the sensor units, placing the fabric-based sensor on top of the flesh.

\section{REFERENCES}

[1] P. Tiezzi, F. Lotti, and G. Vassura, "Polyurethane gel pulps for robotic fingers," in Proc. Int. Conf. Advanced Robotics, vol. 30, 2003.

[2] A. Kadowaki, T. Yoshikai, M. Hayashi, and M. Inaba, "Development of soft sensor exterior embedded with multi-axis deformable tactile sensor system," in Proc. RO-MAN, 2009, pp. 1093-1098.

[3] G. Cannata, M. Maggiali, G. Metta, and G. Sandini, "An embedded artificial skin for humanoid robots," in Proc. Multisensor Fusion and Integration for Intelligent Systems (MFI), 2008, pp. 434-438.

[4] A. Schmitz, U. Pattacini, F. Nori, L. Natale, G. Metta, and G. Sandini, "Design, realization and sensorization of the dexterous iCub hand." in Proc. Humanoids, 2010, pp. 186-191.

[5] O. Kerpa, K. Weiss, and H. Worn, "Development of a flexible tactile sensor system for a humanoid robot," in Proc. IROS, 2003.

[6] K. Kim, K. Lee, et al., "Polymer-based flexible tactile sensor up to $32 \times 32$ arrays integrated with interconnection terminals," Sensors and Actuators A: Physical, vol. 156, no. 2, pp. 284-291, 2009.

[7] H. Alirezaei, A. Nagakubo, and Y. Kuniyoshi, "A highly stretchable tactile distribution sensor for smooth surfaced humanoids," in Proc. Humanoids, 2007, pp. 167-173.

[8] A. Drimus, G. Kootstra, A. Bilberg, and D. Kragic, "Design of a flexible tactile sensor for classification of rigid and deformable objects," Robotics and Autonomous Systems, vol. 62, no. 1, pp. 3 $15,2014$.

[9] J. A. Fishel, "Design and use of a biomimetic tactile microvibration sensor with human-like sensitivity and its application in texture discrimination using bayesian exploration," Ph.D. dissertation, University of Southern California, 2012.

[10] Shadow Robot Company. [Online]. Available: www.shadowrobot.com

[11] G. H. Büscher, R. Kõiva, C. Schürmann, R. Haschke, and H. J. Ritter, "Flexible and stretchable fabric-based tactile sensor," Robotics and Autonomous Systems, vol. 63, no. 3, pp. 244 - 252, 2015, advances in Tactile Sensing and Touch-based Human Robot Interaction.

[12] G. Vásárhelyi, M. Adám, E. Vázsonyi, I. Bársony, and C. Dücsô, "Effects of the elastic cover on tactile sensor arrays," Sensors and Actuators A: Physical, vol. 132, no. 1, pp. 245-251, 2006.

[13] R. Kõiva, M. Zenker, C. Schürmann, R. Haschke, and H. J. Ritter, "A highly sensitive 3d-shaped tactile sensor," in Proc. Advanced Intelligent Mechatronics (AIM), 2013, pp. 1084-1089.

[14] S. Hochreiter and J. Schmidhuber, "Long short-term memory," Neural computation, vol. 9, no. 8, pp. 1735-1780, 1997.

[15] M. Sundermeyer, R. Schlüter, and H. Ney, "LSTM neural networks for language modeling." in INTERSPEECH, 2012. 
[16] F. Weninger, J. Bergmann, and B. Schuller, "Introducing currennt-the munich open-source cuda recurrent neural network toolkit," Journal of Machine Learning Research, vol. 15, 2014.

[17] P. J. Werbos, "Backpropagation through time: what it does and how to do it," Proc. of IEEE, vol. 78, no. 10, pp. 1550-1560, 1990. 